MODELING, IDENTIFICATION AND CONTROL, 1994, VOL. 15, NO. 1, 55-63

doi:10.4173/mic.1994.1.5

\title{
A solution to the problem of constructing a state space model from time series
}

\author{
DAVID DI RUSCIO $\dagger$, ROLF HENRIKSEN $\uparrow$ and JENS G. BALCHEN $\dagger$
}

Keywords: Combined deterministic-stochastic systems; identification; minimal realization; state space modeling; time series.

The problem of constructing minimal realizations from arbitrary input-output time series which are only covariance stationary (not necessarily stationary) is considered. An algorithm which solves this problem for a fairly nonrestrictive class of exogenous (input) signals is presented. The algorithm is based upon modeling nonzero exogenous signals by linear models and including these in the total system model.

\section{Introduction}

The general solution to the problem of constructing minimal realizations from time series is of great importance, not only conceptually but also in many estimation problems, and would be of interest to many who work in the field of system identification and system theory. One particularly important application is the estimation of econometric models, see Aoki (1990, 1991), Östermark and Aoki (1992) and Henriksen (1985). One particular difficulty with constructing minimal realizations of economic time series is that it has to be based upon arbitrary time series (not stylized and simplified ones) which usually cannot be assumed to be stationary, only covariance stationary. The means of all variables involved will generally be nonzero, time varying, and unknown. In order to construct a minimal stochastic realization in accordance with Faurre (1976) or Aoki (1990), such mean values or trends have to be removed in order to compute the autocovariance function of the output.

However, in order to remove these trends in a proper fashion, we need a model of the underlying system, this model is unknown at that stage. As point of fact, if we had this model we could remove all trends and solve the stochastic realization problem which provides us with the said model.

Aoki (1991) and Östermark and Aoki (1992) solve this problem by assuming all expected values to be constant. These values are estimated by using the sample means which enable the removal of all nonzero expectations. However, this approach fails when the mean values are time varying. Di Ruscio and Ljungquist (1992) solved this problem by eliminating trends by taking differences of the correlation matrices. This last method does not work for arbitrary exogenous time series, although it works excellently when the cross-correlation matrices are constant. A method which is more general with respect to the type of exogenous time series is presented in Di Ruscio (1992, 1993). This method has restrictions on the order of the underlying system.

In this paper we resolve the problem by including a linear model of the (nonzero) exogenous variables in our representation. Note that this approach is in accordance

Received 8 October 1993.

$\dagger$ Department of Engineering Cybernetics, Norwegian Institute of Technology, N-7034 Trondheim, Norway.

(C) IEEE. Reprinted, from Proceedings of the 32nd IEEE Conf. Decision and Control, San Antonio, 13-17 December 1993, with kind permission from the IEEE. 
with the behavioural framework introduced by Willems (1986a, b, 1987), where no distinction between inputs and outputs is actually made. This approach is definitely proper in econometric modeling where the entire econometric system of the world can be considered to be autonomous. There will usually be some kind of feedback from socalled outputs to so-called inputs in a submodel, and this can be taken care of by introducing a model of the inputs.

\section{Problem definition and preliminaries}

Assume that the underlying system can be described by a discrete-time, time invariant, linear state space model of the form

$$
\begin{aligned}
x_{i+1} & =A x_{i}+B u_{i}+C e_{i} \\
y_{i} & =D x_{i}+E u_{i}+e_{i}
\end{aligned}
$$

where $i \geqslant 0$ is discrete-time, i.e. an integer, $x \in \Re^{n}$ is the state vector with initial value $x_{0}$, $y \in \Re^{m}$ is the system output, $u \in \Re^{r}$ is the system input, $e \in \mathfrak{R}^{m}$ is an unknown innovations process of white noise, assumed to be covariance stationary, with zero mean and $\mathrm{E}\left(e_{i} e_{i}^{\mathrm{T}}\right)=\Delta . A, B, C, D$ and $E$ are constant matrices of appropriate dimensions, where $(D$, $A)$ is an observable pair.

The problem investigated in this paper is to construct a state space representation of the deterministic part of the system, i.e. the quadruple $(A, B, D, E)$, from known input output time series $u$ and $y$. The matrices $C$ and $\Delta$ can be determined when the deterministic part of the model is known, see Di Ruscio and Ljungquist (1992).

\section{Part I}

The results in this Section are presented in four steps. They are then compactly put together as an algorithm which solves the realization problem. The algorithm also turns out to solve the deterministic realization problem without the need of doing experiments on the process. The most important result in this section is that we show that the minimal realization problem can be solved for a non-restrictive class of input signals. This result is used in $\S 4$.

Step 1 (modeling the input signal)

Assume that the input $u_{i}$ can be described by the model

$$
\begin{aligned}
\zeta_{i+1} & =A_{u} \zeta_{i}+B_{u} \tilde{u}_{i} \\
u_{i} & =D_{u} \zeta_{i}
\end{aligned}
$$

where the external input signal $\tilde{u}_{i}$, for the sake of convenience, is chosen as an impulse at time $i$, a white noise sequence, or a combination of both. $u$ is defined to be exogeneous if not influenced by the state vector in the process model. Note that the results in this paper can be extended to the more general case where, $u_{i}=D_{u} \zeta_{i}+E_{u} \tilde{u}_{i}$.

Step 2 (defining the augmented output)

The process model augmented with the input model is

$$
\begin{gathered}
{\left[\begin{array}{l}
x_{i+1} \\
\zeta_{i+1}
\end{array}\right]=\left[\begin{array}{ll}
A & B D_{u} \\
0 & A_{u}
\end{array}\right]\left[\begin{array}{l}
x_{i} \\
\zeta_{i}
\end{array}\right]+\left[\begin{array}{l}
0 \\
B_{u}
\end{array}\right] \tilde{u}_{i}+\left[\begin{array}{l}
C \\
0
\end{array}\right] e_{i}} \\
\tilde{y}_{i}=\left[\begin{array}{l}
y_{i} \\
u_{i}
\end{array}\right]=\left[\begin{array}{ll}
D & E D_{u} \\
0 & D_{u}
\end{array}\right]\left[\begin{array}{l}
x_{i} \\
\zeta_{i}
\end{array}\right]+\left[\begin{array}{l}
I_{m} \\
0
\end{array}\right] e_{i}
\end{gathered}
$$


where $I_{m}$ is the $m \times m$ identity matrix. Note that the initial values $x_{0}$ can be represented by infinitely fast dynamics and also augmented into the model. This is shown in the Appendix.

Step 3 (impulse responses for the augmented system)

Define

$$
\tilde{\Lambda}_{k}=\mathrm{E}\left(\tilde{y}_{i+k} \tilde{u}_{i}^{\mathrm{T}}\right) \quad \tilde{U}_{k}=\mathrm{E}\left(\tilde{u}_{i+k} \tilde{u}_{i}^{\mathrm{T}}\right) \quad \tilde{Z}_{k}=\mathrm{E}\left(\tilde{x}_{i+k} \tilde{u}_{i}^{\mathrm{T}}\right)
$$

we then find

$$
\begin{aligned}
& \tilde{\Lambda}_{0}=\tilde{D} \tilde{Z}_{0} \\
& \tilde{\Lambda}_{k}=\tilde{D} \tilde{A}^{k} \tilde{Z}_{0}+\sum_{i=1}^{k} \tilde{D} \tilde{A}^{k-1} \tilde{B} \tilde{U}_{i-1} \quad \forall k \geqslant 1
\end{aligned}
$$

If the signal $\tilde{u}_{i}$, which generate $u_{i}$ through the linear model $\left(A_{w}, B_{u}, D_{u}\right)$, is of the type described in Step 1, then we have

$$
\tilde{Z}_{0}=\mathrm{E}\left(\tilde{x}_{i} \tilde{u}_{i}^{\mathrm{T}}\right)=0
$$

and the impulse responses for the augmented system defined by

$$
\tilde{D} \tilde{A}^{k-1} \widetilde{B}=\tilde{H}_{\tilde{y} \tilde{u} k} \quad \forall k \geqslant 1
$$

are determined from

$$
\tilde{H}_{\bar{y} \tilde{u} k} \tilde{U}_{0}=\tilde{\Lambda}_{k}-\sum_{i=1}^{k-1} \tilde{H}_{\tilde{y} \tilde{u}(k-i)} \tilde{U}_{i} \quad \forall k \geqslant 1
$$

if the external signal is sufficiently 'rich' for the inversion of $\tilde{U}_{0}$. The next step is to separate the impulse responses for the quadruple $(A, B, D, E)$ and the triple $\left(A_{u}, B_{u}, D_{u}\right)$ from the impulse responses of the augmented system, $\tilde{H}_{\tilde{y} \tilde{k} k}$.

Step 4 (impulse responses for the system $(A, B, D, E)$

The augmented system matrices $\tilde{A}, \widetilde{B}$ and $\tilde{D}$ can be realized from the Hankel matrix and the shifted Hankel matrix constructed from the sequence $\widetilde{H}_{\text {jük }}$. Note that the matrices $A$ and $A_{u}$ are directly determined from an ordered block real Schur decomposition of the augmented system, i.e. $\tilde{A}_{s}=M^{\mathrm{T}} \tilde{A} M, \widetilde{B_{s}}=M^{\mathrm{T}} \tilde{B}$ and $\widetilde{D_{s}}=\tilde{D} M$, where

$$
\tilde{A}_{s}=\left[\begin{array}{ll}
A_{s 11} & A_{s 12} \\
0 & A_{s 22}
\end{array}\right] .
$$

We can chose $A=A_{s 11}, A_{u}=A_{222}$ if $\tilde{A}_{s}$ is sufficiently ordered. The other matrices can possibly be found by using a procedure similar to the results in Rachid and Hashim (1992) where the Schur decomposition was used in model reduction. However, $D$ and $B$ are in general not unique in this case.

We will now show that the impulse responses for the systems $(A, B, D, E)$ and $\left(A_{u}, B_{u}\right.$,

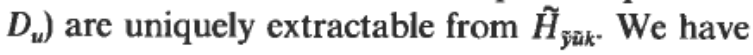

$$
\tilde{H}_{\text {yu } 1}=\left[\begin{array}{r}
E D_{u} B_{u} \\
D_{u} B_{u}
\end{array}\right]
$$

and $E$ is defined if $D_{u} B_{u}$ is invertible. Similarly

$$
\tilde{H}_{\tilde{y} \tilde{u} 2}=\left[\begin{array}{l}
D B D_{u} B_{u}+E D_{u} A_{u} B_{u} \\
D_{u} A_{u} B_{u}
\end{array}\right]
$$


gives the impulse responses $D_{u} A_{u} B_{u}$ and $D B$. Continuing this procedure gives

$$
\tilde{H}_{\tilde{y} \tilde{u} 3}=\left[\begin{array}{l}
D A B D_{u} B_{u}+D B D_{u} A_{u} B_{u}+E D_{u} A_{u}^{2} B_{u} \\
D_{u} A_{u}^{2} B_{u}
\end{array}\right]
$$

and so one. The above results are presented in Algorithm 3.1 below.

Algorithm 3.1. (Determination of impulse responses)

1. Define the cross correlation matrices for the augmented system

$$
\begin{aligned}
& \tilde{U}_{k}=\mathrm{E}\left(\tilde{u}_{i+k} \tilde{u}_{i}^{\mathrm{T}}\right) \\
& \tilde{\Lambda}_{k}=\mathrm{E}\left(\tilde{y}_{i+k} \tilde{u}_{i}^{\mathrm{T}}\right)
\end{aligned}
$$

2. Define the impulse responses for the augmented model.

$$
\tilde{H}_{\hat{y} \tilde{u} k}=\left(\tilde{\Lambda}_{k}-\sum_{i=1}^{k-1} \tilde{H}_{\tilde{y} \tilde{u}(k-i)} \tilde{U}_{i}\right) \tilde{U}_{0}^{-1} \quad \forall k \geqslant 1
$$

Define a partitioned form

$$
\tilde{H}_{\tilde{y} \tilde{u} k}=\left[\begin{array}{l}
H_{y \tilde{u} k}^{\prime} \\
H_{u \ddot{u} k}
\end{array}\right] \text { where } \quad H_{u \tilde{u} k}=D_{u} A_{u}^{k-1} B_{u}
$$

3. The impulse responses for the quadruple $(A, B, D, E)$ are generated recursively as

where

$$
H_{y u(k-1)}=\left(H_{y \tilde{u} k}^{\prime}-\sum_{i=1}^{k-1} H_{y u(k-i)} H_{u \tilde{u}(i+1)}\right) H_{u \tilde{u} 1}^{-1} \quad \forall k \geqslant 1
$$

$$
H_{y u 0}=E \text { and } H_{y u k}=D A^{k-1} B \quad \forall k \geqslant 1 .
$$

\section{Part II}

The results herein are presented in three algorithms for the efficient realization of combined stochastic and deterministic systems. The first is based on cross correlations of the output $y_{i}$ and the output of the augmented system, $\mathrm{E}\left(y_{i+k} \tilde{y}_{i}\right)$, in addition to a realization of the exogenous input. The second is based on the correlations function of the output of the augmented system, $\mathrm{E}\left(\tilde{y}_{i+k} \tilde{y}_{i}\right)$. The third is used to determine $(B, E)$ from given $(D, A)$, e.g. from the two first algorithms.

Algorithm 4.1. (System and augmented output cross correlation)

Step 1. Check for dynamics in the exogenous input. Define the correlation matrices

$$
U_{k}=\mathbf{E}\left(u_{i+k} u_{i}^{\mathrm{T}}\right)
$$

which satisfy the autonomous system

$$
U_{k}=D_{u} A_{u}^{k-1} Z_{\zeta u 1} \quad \text { where } \quad Z_{\zeta u 1}=\mathrm{E}\left(\zeta_{i+1} u_{i}^{\mathrm{T}}\right)
$$

when $\mathrm{E}\left(\tilde{u}_{i+k} u_{i}\right)=0 \forall k \geqslant n s$. Note that we have chosen $n s=1$ for simplicity. Determine a realization for the triple $\left(D_{u}, A_{u}, Z_{\zeta_{u 1}}\right)$.

Step 2. Define the correlation matrices for the output, or the cross correlation matrices between the output and the input, i.e.

$$
\Lambda_{k}=\mathrm{E}\left(y_{i+k} y_{i}^{\mathrm{T}}\right) \text { where } \Lambda_{k}=\tilde{D}_{1} \tilde{A}^{k-1} Z_{\tilde{x} y 1} \text { and } Z_{\tilde{x} y 1}=\left[\begin{array}{l}
\mathrm{E}\left(x_{i+1} y_{i}^{\mathrm{T}}\right. \\
\mathrm{E}\left(\zeta_{i+1} y_{i}^{\mathrm{T}}\right)
\end{array}\right]
$$


or

$$
S_{k}=\mathrm{E}\left(y_{i+k} u_{i}^{\mathrm{T}}\right) \text { where } S_{k}=\tilde{D}_{1} \widetilde{A}^{k-1} Z_{\tilde{x} \mu 1} \text { and } Z_{\tilde{x} \mu 1}=\left[\begin{array}{l}
\mathrm{E}\left(x_{i+1} u_{i}^{\mathrm{T}}\right) \\
\mathrm{E}\left(\zeta_{i+1} u_{i}^{\mathrm{T}}\right)
\end{array}\right]
$$

where $\tilde{D}_{1}=\left[\begin{array}{lll}D & E D_{u}\end{array}\right]$. Note that we could have chosen $S_{k}=\tilde{D}_{1} \tilde{A}^{k} Z_{\tilde{x} u 0}$ if $\mathrm{E}\left(\tilde{u}_{i} u_{i}^{\mathrm{T}}\right)=0$.

Step 3. Define the Hankel matrix and the shifted Hankel matrix and determine a realization for the augmented system $\left(\widetilde{D_{1}}, \widetilde{A}, Z_{\tilde{x} y 1}\right)$ or $\left(\widetilde{D_{1}}, \widetilde{A}, Z_{\tilde{x} u 1}\right)$. Separate $A$ by a block real Schur decomposition of $\widetilde{A}$ such that $A_{u}$ is located in the lower right corner. Determine a block modal form with $A$ and $A_{u}$ on the diagonal, if required. Choose the $D$ matrix as the left block in $\tilde{D}_{1}$.

Step 4 . The matrices $\left(D, A, D_{u}, A_{u}\right)$ are given from the above. Determine a realization for $(A, B, D, E)$ and $\left(A_{u}, B_{u}, D_{u}\right)$ if required. Note that the realization problem is only partly solved at this stage, because $\left(B, E, B_{u}\right)$ is undefined. See Algorithm 4.3 for the determination of $(B, E)$.

Note that analysis based on Equation (26) for the determination of $(D, A)$ can give poor results compared to Equation (25), and is not recommended.

The justification for this statement is explained as follows. Assume that process noise is present in the system. In this case the correlation matrices of the output reflect the dynamics better than cross-correlation between outputs and inputs, because the excitations from the noise in addition to the input excitations are reflected in the correlation matrices. This is also easily seen in the case when the input sequences are zero, and the system dynamics is only excited by process noise. In this case the crosscorrelation matrices are zero, but the correlation matrices contain information about the system dynamics.

Another fact is that the correlation and the cross-correlation matrices give the same information of system dynamics in the deterministic case, i.e. when no process noise is present. However, the cross-correlation matrices between the input and output time series give important information about the input matrix $B$.

The next algorithm is more general than the previous algorithm and turns out to have remarkable numerical properties. Step 1 and 2 in Algorithm 4.1 turn out to be special cases. So we proceed directly, without completing the above, at this stage. However, Step 1 in Algorithm 4.1 should usually be determined prior to the next Algorithm 4.2.

Algorithm 4.2 (Augmented output correlation)

Step 1. Define the augmented system output from given time series $y$ and $u$, i.e.

$$
\tilde{y}_{i}=\left[\begin{array}{l}
y_{i} \\
u_{i}
\end{array}\right]
$$

and compute the correlation matrices

which satisfy

$$
\tilde{\Lambda}_{k}=\mathrm{E}\left(\tilde{y}_{i+k} \tilde{y}_{i}^{\mathrm{T}}\right) \quad \forall k=1, \ldots, 2 K
$$

$$
\begin{gathered}
\tilde{\Lambda}_{k}=\tilde{D} \tilde{A}^{k-1} Z_{\tilde{x} \tilde{y} 1} \\
\tilde{D}=\left[\begin{array}{ll}
D & E D_{u} \\
0 & D_{u}
\end{array}\right] \quad \tilde{A}=\left[\begin{array}{ll}
A & B D_{u} \\
0 & A_{u}
\end{array}\right] \quad Z_{\tilde{x} \tilde{y} 1}=\left[\begin{array}{ll}
\mathrm{E}\left(x_{i+1} y_{i}^{\mathrm{T}}\right. & \mathrm{E}\left(x_{i+1} u_{i}^{\mathrm{T}}\right) \\
\mathrm{E}\left(\zeta_{i+1} y_{i}^{\mathrm{T}}\right) & \mathrm{E}\left(\zeta_{i+1} u_{i}^{\mathrm{T}}\right)
\end{array}\right] .
\end{gathered}
$$

Step 2. Define the Hankel matrix $\Lambda_{K} \in \Re^{K(m+r) \times K(m+r)}$, and the shifted Hankel matrix $\Lambda_{A} \in \Re^{K(m+r) \times K(m+r)}$, from $\tilde{\Lambda}_{k} \forall k=1, \ldots, 2 K$ and determine a realization for the triple $\left(\tilde{D}, \tilde{A}, Z_{\bar{x} \tilde{y} 1}\right)$, say $\left(\tilde{D}^{b}, \widetilde{A}^{b}, Z_{\dot{x y} 1}^{b}\right)$. Reorder $\widetilde{A}^{b}$ by a block real Schur decomposition such that $A$ is located in the upper left corner, if required. 
Step 3. The matrices $\left(D, A, D_{u}, A_{u}\right)$ are determined directly from Step 2. Determine a realization for $(A, B, D, E)$ and $\left(A_{u}, B_{u}, D_{u}\right)$ if required. $(B, E)$ is determined from Algorithm 4.3.

Note that the lower right block in $\tilde{D}$ will always be zero when $u$ is not influenced by the state vector $x$, i.e. when $u$ is exogenous.

It is not trivial to determine equations that define the $(B, E)$ matrices. One approach is to compare submatrices in the estimated realization $\left(\tilde{D}^{b}, \widetilde{A}^{b}, Z_{\tilde{x} \tilde{1} 1}^{b}\right)$ with those of $(\tilde{D}, \tilde{A}$, $\left.Z_{\tilde{x} \tilde{y} 1}\right)$. However, this approach is generally not unique.

Several methods for the determination of the $(B, E)$ matrices are possible when the pair $(D, A)$ are known. We will in what follows present one fairly general method, and then discuss some special cases of this method.

The matrices $B$ and $E$ satisfy

$$
\begin{aligned}
Z_{k+1} & =A z_{k}+B U_{k} \\
S_{k} & =D Z_{k}+E U_{k}
\end{aligned}
$$

where $Z_{k}=\mathrm{E}\left(x_{i+k} u_{i}^{\mathrm{T}}\right)$ and $U_{k}=\mathrm{E}\left(u_{i+k} u_{i}\right)$. When $(D, A)$ is an observable pair, and $(D, A$, $\left.Z_{0}\right)$ are given, we can solve these equations with respect to $(B, E)$.

The following iterative procedure, Algorithm 4.3, turns out to be quite effective in solving this problem. A least squares approach directly on $S_{k}=\mathrm{E}\left(y_{i+k} u_{i}\right) \forall k=0, \ldots, L$ is sufficient in the single input case. This will be discussed after the presentation of the general algorithm.

\section{Algorithm 4.3 (Determination of $(B, E)$ from given $(D, A)$ )}

Step 1. Specify $(D, A)$ and an initial matrix $Z_{0}^{p}$ from the previous algorithms. Set $p:=0$, where $p$ is the index of iteration.

Step 2. Solve the following equations with respect to $E^{p}$ and $B^{p}$.

$$
\begin{aligned}
E^{p} & =\left(S_{0}-D Z_{0}^{p}\right) U_{0}^{-1} \\
H_{k} & =\left(S_{k}-D A^{k} Z_{0}^{p}-E^{p} U_{k}-\sum_{i=1}^{k-1} H_{k-i} U_{i}\right) U_{0}^{-1} \quad \forall k=1, \ldots, L \\
B^{p} & =\left(O^{\mathrm{T}} O\right)^{-1} O^{\mathrm{T}} H_{B}
\end{aligned}
$$

where $H_{B}^{\mathrm{T}}=\left[H_{1}^{\mathrm{T}} H_{2}^{\mathrm{T}} \ldots H_{L}^{\mathrm{T}}\right]$ and $O \in \Re^{L m \times n}$ is the observability matrix for the pair $(D, A)$. $U_{0}=\mathrm{E}\left(u_{i} u_{i}^{\mathrm{T}}\right)$ is assumed to be non-singular.

Step 3. Simulate the system $\left(D, A, B^{p}, E^{p}\right)$ with specified initial values (usually $x_{0}=0$ ) and generate new values $y_{i}^{p}, x_{i}^{p}$. Update $Z_{0}$ and $S_{k}$ from

$$
Z_{0}^{p+1}=\mathrm{E}\left(x_{i}^{p} u_{i}^{\mathbf{T}}\right) \quad S_{k}^{p+1}=\mathrm{E}\left(y_{i+k}^{p} u_{i}^{\mathrm{T}}\right) \forall k=0, \ldots, L
$$

Step 4. Check for convergence. If the norm of the matrix

$$
J_{c}=\frac{1}{L+1} \sum_{k=0}^{L}\left(S_{k}-S_{k}^{p+1}\right)
$$

is greater than a specified $\epsilon$, then set $p:=p+1$ and return to Step 2, else, terminate.

Simulation experiments have shown that, when $A$ is stable, we have convergence in the sense that $J_{c}$ is minimized, i.e. $J_{c} \rightarrow 0$. We have also experienced that we can arrive at a solution that is different from the underlying $(B, E)$ matrices, even when $J_{c} \rightarrow 0$. This 
indicates that the matrices $(B, E)$ are generally not unique. The Algorithm 4.3 is found to work for systems which have nonminimum phase behaviour from the input to the output. Algorithms 4.1, 4.2 and 4.3 are in combination the solution to the problem of constructing a realization of the deterministic part of the system, i.e. the quadruple $(A, B, D, E)$.

The general Algorithm 4.3 is based in iterations. Therefore, it will be of interest to discuss some special cases, for the determination of $(B, E)$ from given $(D, A)$, where iterations are not necessary.

Two cases are of particular interest. The first is the case where the number of states $n$ is equal to the number of outputs $m$. A simple least squares algorithm for the determination of the quadruple $(A, B, D, E)$ is discussed in Di Ruscio (1993). This approach can easily be modified to a procedurc for the determination of $(B, E)$ from given $(D, A)$.

A second special case is the single input case, which will be presented below. In this case $U_{k}$ is a scalar, and we have

$$
\left[\begin{array}{l}
\bar{S}_{1} \\
\bar{S}_{2} \\
\vdots \\
\bar{S}_{k}
\end{array}\right]=\left[\begin{array}{ll}
D \bar{A} & D U_{0} \\
D \bar{A}^{2} & D A U_{0}+D U_{1} \\
\vdots & \vdots \\
D \bar{A}^{k} & D A^{k-1} U_{0}+D A^{k-2} U_{1}+\ldots+D U_{k-1}
\end{array}\right]\left[\begin{array}{l}
Z_{0} \\
B
\end{array}\right]
$$

where $\bar{S}_{j}=S_{j}-S_{0} U_{0}^{-1} U_{j}$ and $D \bar{A}^{j}=D A^{j}-D U_{0}^{-1} U_{j} \forall j=1, \ldots, k$, which define $Z_{0}$ and $B$ from a least squares solution, and $E=\left(S_{0}-D Z_{0}\right) U_{0}^{-1}$ (provided $U_{0}$ is invertible). Note that the above also can be written as

$$
\left[\begin{array}{l}
S_{1} \\
S_{2} \\
\vdots \\
S_{k}
\end{array}\right]=\left[\begin{array}{lll}
D A & D U_{0} & U_{1} \\
D A^{2} & D A U_{0}+D U_{1} & U_{2} \\
\vdots & \vdots & \vdots \\
D A^{k} & D A^{k-1} U_{0}+D A^{k-2} U_{1}+\ldots+D U_{k-1} & U_{k}
\end{array}\right] \quad\left[\begin{array}{l}
Z_{0} \\
B \\
E
\end{array}\right] .
$$

However, Eqn. (37) and $E=\left(S_{0}-D Z_{0}\right) U_{0}^{-1}$ is usually better numerically condition for the determination of $\left(Z_{0}, B, E\right)$ than the latter expression.

Note that when $E$ is known to be zero, then the above equations should be modified to take this into consideration. In this case, $E$ should also be set to zero in Algorithm 4.3. This is because small deviations in $E$ from zero can lead to large errors in transmission zeroes, and wrong conclusions can be drawn with respect to phase behaviour. Even more important, the determination of $E$ in addition to $\left(Z_{0}, B\right)$ requires that the input signal $u$ is more 'rich' compared to the determination of only $\left(Z_{0}, B\right)$. When $E=0,(37)$ should be modified by substituting $\bar{S}_{j}$ with $S_{j}$ and $D \bar{A}^{j}$ with $D A^{j}$. Note also that the solution $Z_{0}$ from this least squares method can be used as initial value to the Algorithm 4.3 to refine the solution further.

\section{A numerical example}

We will illustrate the method on the same example as in Di Ruscio and Ljungquist (1992).

$$
\begin{aligned}
& A=\left[\begin{array}{rr}
1.5 & 1.0 \\
-0.7 & 0
\end{array}\right] \quad B=\left[\begin{array}{r}
2.0 \\
-1.3
\end{array}\right] \quad C=\left[\begin{array}{l}
0.5 \\
1.5
\end{array}\right] . \\
& D=\left[\begin{array}{ll}
1 & 0
\end{array}\right] \\
& E=0
\end{aligned}
$$


Table 1. Simulation results with $N=4000$ samples and $L=10$.

\begin{tabular}{|c|c|c|c|c|c|c|c|c|}
\hline \multicolumn{3}{|c|}{ System } & \multirow{2}{*}{$\frac{\text { Alg. }}{K}$} & \multicolumn{5}{|c|}{ Realization } \\
\hline $\mathbf{u}$ & $\Delta$ & $S / N$ & & $\lambda(\hat{A})$ & $H_{d}(1)$ & $\hat{p}_{d}$ & $J_{c}$ & $\operatorname{dim} A_{u}$ \\
\hline$u_{1}$ & 0 & $\infty$ & 15 & $0.750 \pm \mathrm{j} 0.371$ & 3.49 & 0.651 & 0.005 & 2 \\
\hline$u_{1}$ & 1 & $0 \cdot 30$ & 15 & $0-738 \pm \mathrm{j} 0-381$ & 3.47 & 0.613 & $0-005$ & 2 \\
\hline$u_{2}$ & 0 & $\infty$ & 15 & $0.750 \pm \mathrm{j} 0.371$ & 3.49 & 0.651 & 0.004 & 10 \\
\hline$u_{2}$ & 1 & $0-30$ & 15 & $0.762 \pm \mathrm{j} 0.365$ & 3.69 & 0.700 & 0.092 & 10 \\
\hline \multicolumn{3}{|c|}{ True system } & & $0.750 \pm \mathrm{j} 0.371$ & $3 \cdot 5$ & 0.65 & 0 & \\
\hline
\end{tabular}

Algorithm 4.2 is used to estimate $(D, A)$ and Algorithm 4.3 is used to estimate $B$. The following input sequences were tried out in the simulations.

$$
\begin{aligned}
& u_{1}(i)=\sin (i) \\
& u_{2}(i)=0-2\left(\sin \left(\frac{i}{25}\right)+\sin \left(\frac{i}{10}\right)+\sin \left(\frac{i}{5}\right)+\sin (i)\right) .
\end{aligned}
$$

The white noise innovation process $e$ was generated with the MATLAB function rand. A normal distribution with seed zero was chosen as input to $r$ and.

The cross correlation matrices between two finite time series $y$ and $u$, for time lag $k$, was computed according to the unbiased estimate, viz.

$$
\mathrm{E}\left(y_{i+k} u_{i}^{\mathrm{T}}\right)=\frac{1}{N-1-k} \sum_{i=0}^{N-k-1} y_{i+k} u_{i}^{\mathrm{T}} \quad k \geqslant 0
$$

where $N$ is the number of samples in the time series.

The results are shown in Table 1, where the following notations are used: The eigenvalues of a square matrix $A$ are denoted $\lambda(A)$. The transfer function from $u$ to $y$ is $H_{d}(z)=D(z I-A)^{-1}+E$. The steady state gain and the zeroes of $H_{d}(z)$ are denoted $H_{d}(1)$ and $p_{d}$, respectively. $S / N$ is the signal-to-noise ratio.

The Table shows that the results from the algorithms in this work are significantly improved compared to the results presented in Example 1 in Di Ruscio and Ljungquist (1992), for these types of input signals. We will argue that the algorithms in Östermark and Aoki (1992) and Di Ruscio and Ljungquist (1992), do not work for this type of input signals, Eqns. (41) and (42).

\section{Conclusions}

The algorithms presented in this paper are from numerous examples found to have remarkable numerical properties for the minimal realization of the quadruple $(A, B, D, E)$ from time series.

An important observation is that the correlations $\mathrm{E}\left(y_{i+k} y_{i}^{\mathrm{T}}\right), \mathrm{E}\left(y_{i+k} u_{i}^{\mathrm{T}}\right)$ and $\mathrm{E}\left(u_{i+k} y_{i}^{\mathrm{T}}\right)$ contain dynamics of the system that generated the exogenous inputs $u_{i}$ in addition to the dynamics from $u$ to $y$. The dynamics of the exogenous variables can be identified from correlation analysis of $\mathrm{E}\left(u_{i+k} u_{i}^{\mathrm{T}}\right)$. The latter observation must be taken into consideration in order for the system dynamics to be properly identified. This fact seems to be neglected in algorithms reported in the literature.

\section{ACKNOWLEDGMENT}

This research has been sponsored by Norske Skog Teknikk and the Royal Norwegian Council for Scientific and Industrial Research (NTNF). 


\section{APPENDIX}

(Inclusion of initial values in the augmented model)

The process model augmented with the input model and the initial values is

$$
\begin{aligned}
& {\left[\begin{array}{l}
x_{i+1} \\
\zeta_{i+1} \\
\chi_{i+1}
\end{array}\right]=\left[\begin{array}{lll}
A & B D_{u} & A \\
0 & A_{u} & 0 \\
0 & 0 & 0
\end{array}\right] \quad\left[\begin{array}{l}
x_{i} \\
\zeta_{i} \\
\chi_{i}
\end{array}\right]+\left[\begin{array}{ll}
0 & 0 \\
B_{u} & 0 \\
0 & A x_{0}
\end{array}\right] \quad\left[\begin{array}{l}
\tilde{u}_{i} \\
\tilde{v}_{i}
\end{array}\right]+\left[\begin{array}{l}
C \\
0 \\
0
\end{array}\right] e_{i}} \\
& \tilde{y}_{i}=\left[\begin{array}{l}
y_{i} \\
u_{i}
\end{array}\right]=\left[\begin{array}{lll}
D & E D_{u} & D \\
0 & D_{u} & 0
\end{array}\right] \quad\left[\begin{array}{l}
x_{i} \\
\zeta_{i} \\
\chi_{i}
\end{array}\right]+\left[\begin{array}{ll}
0 & D x_{0} \\
0 & 0
\end{array}\right] \quad\left[\begin{array}{l}
\tilde{u}_{i} \\
\tilde{v}_{i}
\end{array}\right]+\left[\begin{array}{l}
I_{m} \\
0
\end{array}\right] e_{i}
\end{aligned}
$$

where $\tilde{x}_{0}=0$ and $\tilde{v}_{i}$ is an impulse at time instant $i=0$. Another possibility is simply to remove the state $\chi$. We have

$$
\begin{gathered}
{\left[\begin{array}{l}
x_{i+1} \\
\zeta_{i+1}
\end{array}\right]=\left[\begin{array}{ll}
A & B D_{u} \\
0 & A_{u}
\end{array}\right] \quad\left[\begin{array}{l}
x_{i} \\
\zeta_{i}
\end{array}\right]+\left[\begin{array}{l}
0 \\
B_{u}
\end{array}\right] \tilde{u}_{i}+\left[\begin{array}{l}
A x_{0} \\
A_{u} \zeta_{0}
\end{array}\right] \tilde{v}_{i}+\left[\begin{array}{l}
C \\
0
\end{array}\right] e_{i}} \\
\tilde{y}_{i}=\left[\begin{array}{l}
y_{i} \\
u_{i}
\end{array}\right]=\left[\begin{array}{ll}
D & E D_{u} \\
0 & D_{u}
\end{array}\right] \quad\left[\begin{array}{l}
x_{i} \\
\zeta_{i}
\end{array}\right]+\left[\begin{array}{c}
D x_{0} \\
D_{u} \zeta_{0}
\end{array}\right] \tilde{v}_{i}+\left[\begin{array}{c}
I_{m} \\
0
\end{array}\right] e_{i} \\
\text { REFERENCES }
\end{gathered}
$$

\section{REFERENCES}

AoKI, M. (1990). State Space Modeling of Time Series. Second, revised and enlarged edition (Springer-Verlag, Berlin, Heidelberg).

Аокı, M. (1991). Two complimentary representations of multiple time series in state space innovation forms, invited paper presented at Seminar on Recent Advances on Time Series Analysis and their Impact on Economic Forecasting, Madrid, Spain, December 16-18, 1991.

Di Ruscio, D. (1993). Topics in model based control with applications to the thermo mechanical pulping process, Dr. Ing. thesis. Report no. 93 46-W, Department of Engineering Cybernetics, Norwegian Institute of Technology, N-7034 Trondheim, Norway.

Di Ruscio, D. (1992). A method for the identification of state space models, Report no. 92-116W. Department of Engineering Cybernetics, Norwegian Institute of Technology, N7034 Trondheim.

Di Ruscio, D. and LJUNGQUIST, D. (1992). State space model realization from input-output time series, Report no. 92-106-W. Department of Engineering Cybernetics, Norwegian Institute of Technology, N7034 Trondheim.

FAURRE, P. L. (1976). Stochastic realization algorithms, In System Identification: Advances and Case Studies, eds. R. K. Mehra and D. G. Lainiotis (Academic Press).

HenRiKsen, R. and Di Ruscio, D. (1993). Construction of minimal realizations from arbitrary time series, Twelfth IASTED International Conference, Modeling, Identification and Control. Innsbruck, February 15-17, 1993.

HENRIKSEN, R. (1985). Estimation of large scale implicit models using two-stage methods, Modeling, Identification and Control, 6, 3-19.

RACHID, A. and HASHIM, G. (1992). Model reduction via Schur decomposition, IEEE Trans. on Automatic Control, 37, 666-668.

ÖSTERMARK, R. and AOKI, M. (1992). Time series evidence of impacts of the U.S. economy on the scandinavian economy (by state space modeling), IF AC workshop on economic time series analysis and system identification, Vienna July 1-3, 1992.

Willems, J. C. (1986a). From time series to linear systems--Part I. Finite dimensional linear time invariant systems, Automatica, 22, 561-580.

WiLLEMS, J. C. (1986b). From time series to linear systems-Part II. Exact modelling, Automatica, 22, 675-694.

Willems, J. C. (1987). From time series to linear systems-Part III. Approximate modelling, Automatica, 22, 675-694. 